\title{
Caractérisation électrique des interfaces métal-semiconducteur
}

\author{
C. Barret, G. N. Lu et T. Neffati \\ Institut d'Electronique Fondamentale, UA 22 CNRS, Université Paris-Sud, bâtiment 220, 91405 Orsay Cedex, \\ France
}

(Reçu le 16 janvier 1987, accepté le 14 mai 1987)

\begin{abstract}
Résumé. - Nous présentons un panorama des caractérisations électriques des interfaces métal-semiconducteur. Nous montrons que la nécessité d'effectuer des mesures de barrière précises implique une amélioration des interprétations des anomalies des caractéristiques $I-V$ et $C-V$ des diodes réelles. D'autre part, l'étude des états d'interface par Spectroscopie de Capacité Schottky met en évidence l'existence d'états bien localisés énergétiquement mais à profondeur de pénétration significative dans le semiconducteur. Une modélisation développée à partir de ces deux points permet d'interpréter de façon très simple les principaux comportements réels des caractéristiques électriques.
\end{abstract}

\begin{abstract}
An overview of the electrical characterization of metal-semiconductor interfaces is given. The need of an improvement in the accuracy of the barrier height measurements implies a better understanding in the non-idealities encountered on the $I-V$ and $C-V$ curves. On the other hand, the study of interface states by Schottky Capacitance Spectroscopy shows the existence of states energetically localized in the band gap and having a significant depth of penetration in the semiconductor. A model is developed from these points and provides a new way of interpretation of the main anomalous behaviours of the real electrical characteristics.
\end{abstract}

\section{Introduction.}

Pendant de nombreuses années le seul moyen expérimental d'étude des interfaces M-S a été la mesure de la barrière de potentiel par voie électrique. A la fin des années 70 , une évolution très nette s'est dessinée en faveur de l'étude des surfaces semiconductrices recouvertes de couches métalliques extrêmement minces. Les méthodes électriques ont alors été délaissées au profit des techniques de caractérisation des surfaces. Malgré ou peut-être à cause de l'important acquis apporté par ces nouvelles techniques, on ressent actuellement un besoin de réactualiser les anciens moyens de caractérisation électrique et d'en développer de nouveaux.

Nous montrons d'abord (paragraphe 2) sur quelques exemples la nécessité de déterminer avec une très grande précision la hauteur de barrière pour valider ou rejeter certaines interprétations théoriques. Cette mesure est généralement perturbée par l'existence de non-idéalités dans le comportement électrique des diodes Schottky réelles. Nous discuterons des explications proposées pour ces non-idéalités (paragraphe 3) et montrerons que si certains résultats expérimentaux s'accommodent d'une inter- prétation simple (courant non thermoionique, hauteur de barrière non uniforme...) cela est loin de représenter la majorité des cas.

Le second point est la nécessité d'obtenir des informations sur les états électroniques d'interface dont le rôle prépondérant dans le comportement de la jonction est unanimement reconnu. Nous rappelons au paragraphe 4 quelques résultats obtenus par la méthode de Spectroscopie de Capacité Schottky (SCS) qui permet à partir de mesures de la capacité de jonction sous polarisation directe la détermination des principaux paramètres (énergie, densité, section efficace de capture) de ces états. Un attrait particulier de cette méthode est qu'elle peut s'appliquer à toute diode Schottky, quel que soit le mode de préparation de la surface. Des corrélations peuvent alors être obtenues entre la distribution des états d'interface, la technologie de préparation et les caractéristiques électriques de la diode.

Les résultats obtenus permettent d'esquisser un modèle réaliste (paragraphe 5) de la forme de la barrière de potentiel dans la zone interfaciale en tenant compte d'une pénétration significative des états dans le semiconducteur. Ce modèle peut ensuite être appliqué (paragraphe 6) pour simuler 
les caractéristiques $I-V$ et $C-V$. Nous montrons que même les configurations les plus simples font apparaître les principaux caractères « anormaux » observés sur les courbes réelles.

\section{Hauteurs de barrière.}

La hauteur de barrière $\phi_{\mathrm{B}}$ est la caractéristique essentielle d'un contact métal-semiconducteur. La quasi-totalité des modèles développés pour interpréter le comportement de ces contacts fait appel à des recherches de corrélation entre la valeur de $\phi_{\mathrm{B}}$ et tel ou tel paramètre lié à l'un ou à l'autre des deux matériaux [1-5]. Il est évident que ces corrélations n'ont de sens que si $\phi_{\mathrm{B}}$ est connue avec précision. Deux exemples donnés sur les figures 1 et 2 illustrent la validité pour le moins douteuse de certaines idées pourtant souvent considérées comme définitivement acquises. Le premier exemple porte sur les interfaces M-Si pour lesquelles on estime usuellement qu'une loi de type Cowley et Sze [1] s'applique, c'est-à-dire que la barrière de Schottky varie linéairement avec le travail de sortie du métal. Or les résultats compilés par Freeouf [6] et reproduits sur la figure 1a montrent qu'il y a en fait une dispersion en fonction de la méthode de mesure utilisée bien supérieure à la variation observée d'un métal à l'autre. Le second

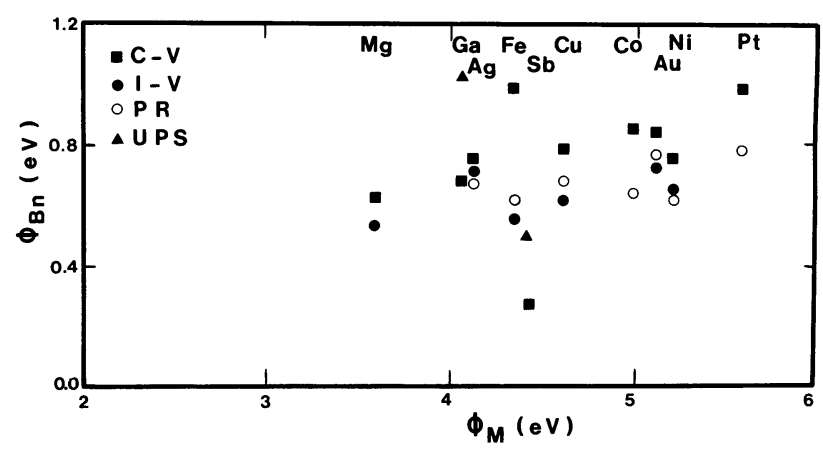

Fig. 1. - Compilation de mesures de hauteur de barrière sur silicium clivé en fonction du travail de sortie du métal et de la méthode de mesure (d'après Freeouf [6]).

[Barrier height on cleaved silicon as a function of metal work function (after Freeouf [6]).]

exemple porte sur les interfaces M-InP. Il est généralement admis à la suite des travaux de Williams [7] et Brillson [8] que deux familles d'interfaces sont à considérer: les interfaces non réactifs qui donnent une barrière de $0,5 \mathrm{eV}$ environ et les interfaces réactifs qui donnent des contacts ohmiques. La figure 2 reproduit des résultats obtenus récemment par Spicer [9] qui montrent au contraire l'absence de corrélation entre hauteur de barrière et chaleur de réaction du métal avec le semiconducteur.

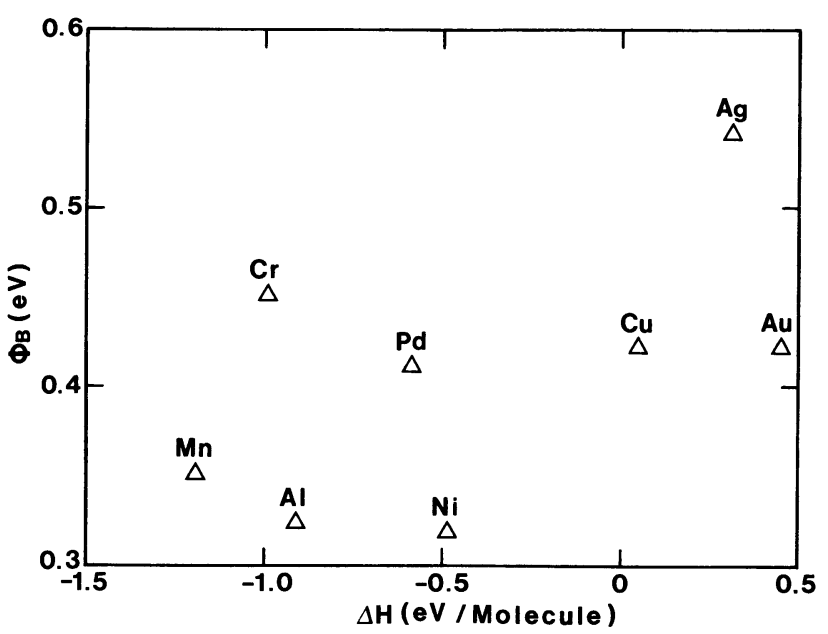

Fig. 2. - Hauteurs de barrière sur InP clivé en fonction de la chaleur de formation du phosphide correspondant (d'après Kendelewicz [9]).

[Barrier height on cleaved InP as a function of the predicted enthalpies of metal-phosphide formation (after Kendelewicz [9]).]

Dans le cas de GaAs un problème légèrement différent mais nécessitant également une détermination très précise de $\phi_{\mathrm{B}}$ se pose. Les expériences de photoémission portant sur des surfaces recouvertes de dépôts métalliques submonocouche $[10,11]$ ont fait apparaître pour plusieurs métaux un écart appréciable de la position d'ancrage du niveau de Fermi suivant le type $n$ ou $p$ du semiconducteur. Si cet écart est conservé pour un métal épais on doit alors trouver une somme des barrières inférieure au gap. Dans le cas contraire cela signifie que la hauteur de barrière n'est pas complètement définie par le dépôt d'une couche métallique monoatomique comme le suppose le modèle de Spicer [12].

\section{Anomalies des caractéristiques électriques.}

Il apparaît clairement d'après la figure 1 que l'obtention de valeurs expérimentales de $\phi_{\mathrm{B}}$ fiables passe par une critique des méthodes de mesure employées. Nous ne discuterons pas ici des problèmes liés aux méthodes optiques pour lesquels nous renvoyons le lecteur aux publications existantes [13, 14]. La grande majorité des mesures publiées utilisent les méthodes électriques classiques $I-V$ et $C-V$. Rappelons qu'idéalement une diode Schottky doit présenter en polarisation directe une caractéristique $\ln$ $I-V$ linéaire de pente $k T / q$ et que l'extrapolation $I_{\mathrm{s}}$ de cette droite à $V=0$ permet d'obtenir la hauteur de barrière par

$$
\Phi_{\mathrm{B}}=k T \ln \frac{S A^{*} T^{2}}{I_{\mathrm{s}}}
$$

où $S$ est l'aire de jonction et $A^{*}$ la constante de 
Richardson modifiée. Dans le cas de la méthode $C-V$ appliquée à une diode réalisée sur substrat à dopage homogène, la caractéristique $C^{-2}-V$ doit être une droite. Sa pente permet de déterminer le dopage du semiconducteur et l'extrapolation $V_{\mathrm{D}_{0}}$ de $V$ à $C^{-2}=0$ donne le potentiel de diffusion, directement relié à la barrière de Schottky. Or ces comportements idéaux ne sont que rarement vérifiés sur des diodes Schottky réelles. La figure 3 montre à titre d'exemple trois types d'anomalies de comportement des caractéristiques $\ln I-V$. Le premier cas est celui

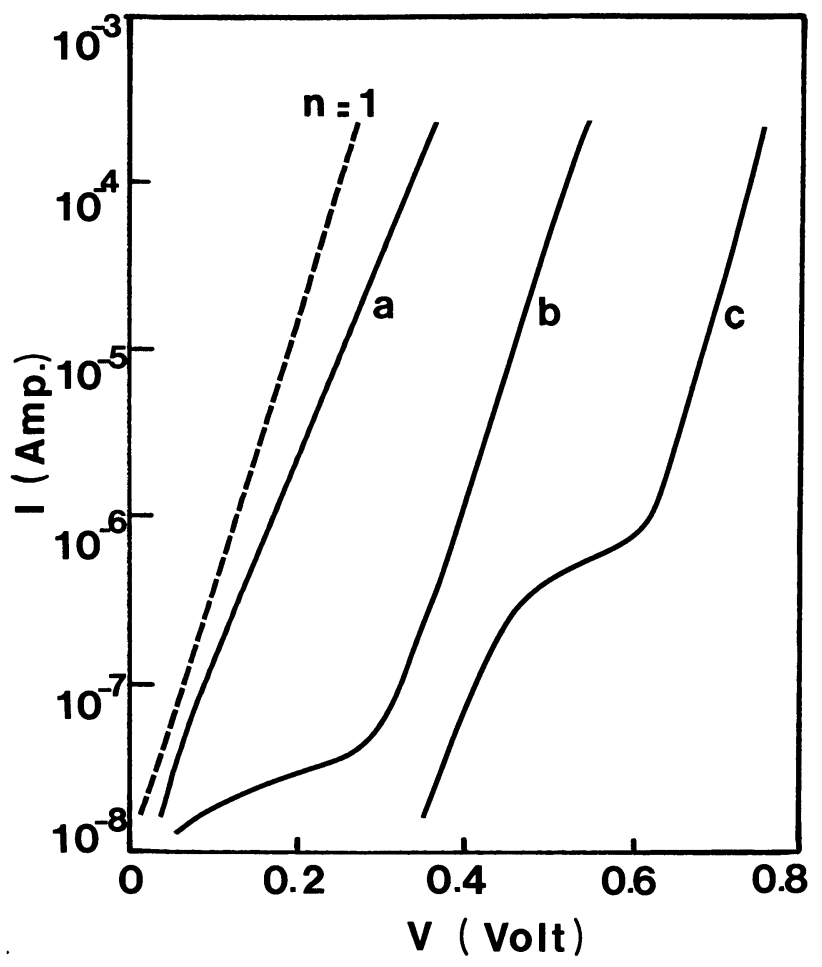

Fig. 3. - Exemples typiques d'anomalies de comportement des caractéristiques $I-V$ d'une diode Schottky: a) coefficient de non-idéalité $n>1$, b) apparence de courant excédentaire à faible polarisation, c) caractéristique « en $\mathbf{N}$ ».

[Typical examples of anomalous behaviour in the $I-V$ curve of a Schottky diode : a) non-ideality coefficient $n>1$, b) appearance of an excess current at low bias voltage, c) « $N$ shaped » characteristic.]

d'une droite mais dont la pente est nettement supérieure à $k T / q$. On a l'habitude de chiffrer le rapport entre la pente réelle et la pente théorique par un paramètre $n$ dit de «non-idéalité ». En fait ce cas où $n$ est supérieur à 1 mais indépendant de $V$ est assez rare. Beaucoup plus fréquemment on observe des ruptures de pente comme sur la courbe $2 b-$ qui s'interprète souvent en admettant la présence d'un courant excédentaire à faible polarisation - ou sur la courbe $2 c$ qui illustre un type classique de nonidéalité appelé «caractéristique en $\mathrm{N}$ » [15].
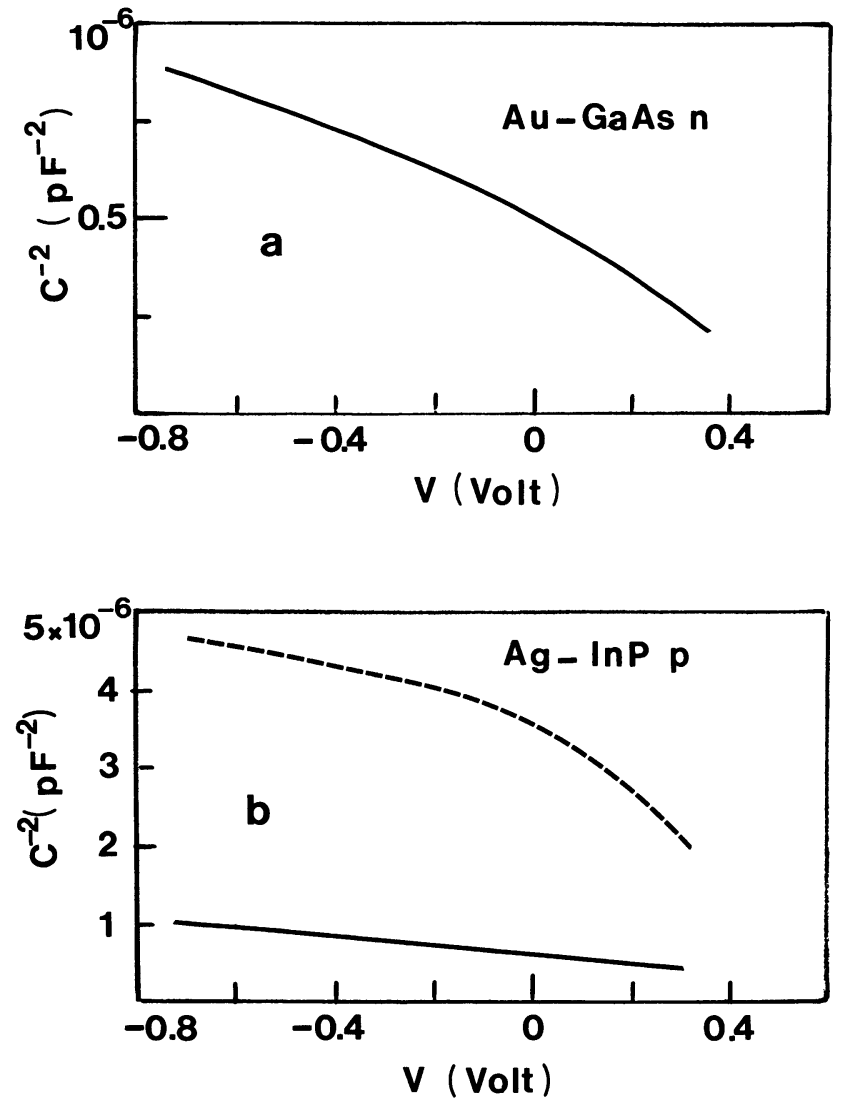

Fig. 4. - Exemples de caractéristiques $C-V$ anormales : a) diode $\mathrm{Au}-\mathrm{GaAs} \mathrm{N}$ clivé sous ultra-vide, b) diodes $\mathrm{Ag}$ InP P clivé sous ultra-vide (-) et à l'air (...).

[Examples of anomalous $C-V$ curves : a) Au-GaAs $\mathrm{N}$ UHV cleaved, b) Ag-InP P UHV cleaved (-) and air cleaved (...).]

Sur la figure 4 sont reportés des exemples caractéristiques d'anomalies de comportement de courbes $C^{-2}-V$. La courbe 4 a est relative à une interface AuGaAs clivée sous ultra-vide et on constate sa nonlinéarité bien que le semiconducteur utilisé soit à dopage homogène. La figure $4 \mathrm{~b}$ compare des résultats obtenus pour Ag-InP soit par clivage sous ultravide soit par clivage à l'air. Dans le second cas une très forte non-linéarité apparaît, quantitativement inexplicable par la présence d'une couche d'oxyde interfaciale d'épaisseur réaliste.

Il est tout à fait évident que des valeurs de $\phi_{\mathrm{B}}$ déduites des courbes présentées sur les figures 3 et 4 ne correspondent pas à la hauteur de barrière réelle. Deux attitudes sont alors possibles. La première consiste à ne conserver comme fiables et à ne considérer pour tester telle ou telle théorie que des mesures vérifiant des critères sévères d'idéalité. Ces critères doivent être : i) $\ln I-V$ linéaire avec $n<1,05$. ii) $C^{-2}-V$ linéaire avec une pente donnant un dopage cohérent avec celui déduit de la résistivité. iii) Hauteur de barrière identique par les deux méthodes à quelques centièmes d'eV près. Il est de 
plus très souhaitable que ces critères soient vérifiés sur une gamme de température aussi large que possible. Enfin une étude systématique de couples métal donné-semiconducteur $\mathrm{n}$ et semiconducteur $\mathrm{p}$ ne peut qu'aider à établir une base de données qui offre toute garantie.

La seconde attitude consiste à expliquer les anomalies constatées et à tenter d'extraire des hauteurs de barrière exactes après correction des courbes expérimentales. Il est naturellement impossible de traiter ici cette question de façon exhaustive. Nous nous limiterons à quelques remarques et à quelques exemples. La première remarque est qu'il n'existe probablement pas d'explication unique et globale de toutes les anomalies rencontrées. La seconde est qu'a priori le problème des anomalies de $I-V$ et celui des anomalies de $C-V$ sont dissociés. En ce qui concerne les courbes $I-V$ trois types d'interprétations sont possibles. La première consiste à invoquer l'existence de courants non thermoioniques [16] (diffusion, recombinaison, tunnel) pour expliquer soit l'existence d'un coefficient $n$ supérieur à 1 soit la présence d'un courant excédentaire. Il est indispensable pour valider ce type d'interprétation que d'une part l'intensité du courant attribué à un tel effet soit compatible avec des paramètres réalistes et que d'autre part le comportement en fonction de la température de ce courant excédentaire soit étudié. La figure 5 montre par exemple des résultats obtenus

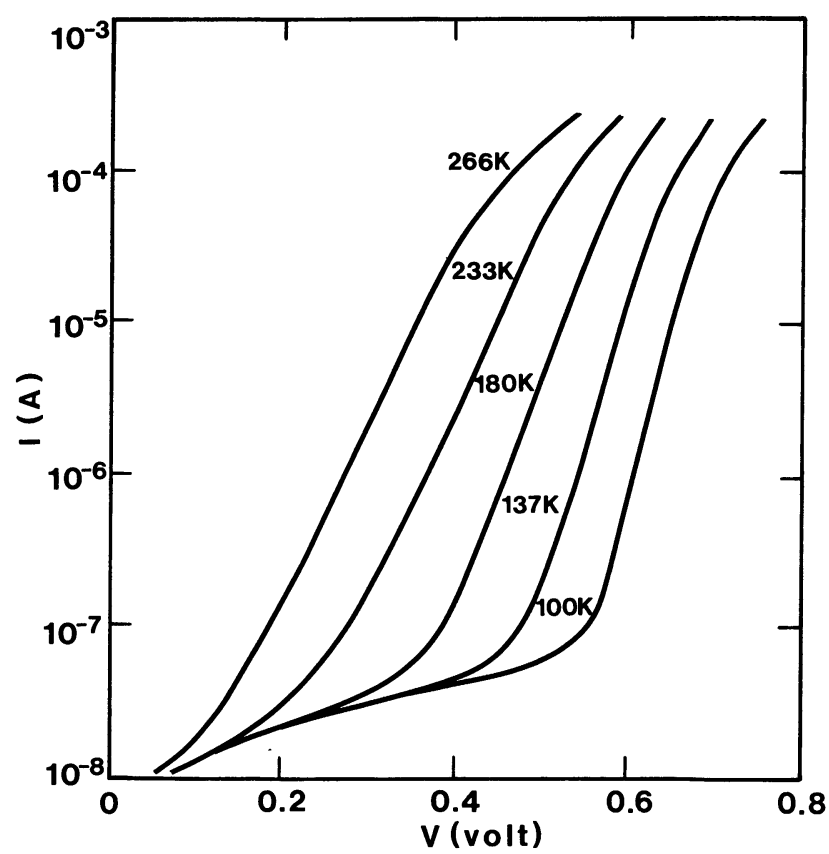

Fig. 5. - Exemple de caractéristique $I-V(A u-I n P p)$ où la déformation à faible polarisation est réellement due à un courant excédentaire non thermoionique [15].

[Example of $I-V$ curve (on Au-InP p) where the anomalous behaviour at low bias voltage is really due to a non thermionic current [15].] sur Au-InP du type $p$ pour lesquels la très faible sensibilité à la température du courant excédentaire incite à l'attribuer à un phénomène d'effet tunnel [15]. Le second type d'explication suppose une dépendance de la hauteur de barrière à la tension appliquée. Cette idée a été développée en particulier par Card et Rhoderick [17] qui calculent la valeur de $n$ associée à la chute de tension à travers une couche isolante interfaciale. S'il est très probable que ce modèle soit valide pour des structures de type MIS tunnel ayant une couche d'oxyde de plusieurs dizaines d'angströms il n'est pas applicable directement aux contacts intimes. Nous montrerons cependant au paragraphe 6 que l'idée de base peut être reprise pour des interfaces idéaux sans oxyde et permet d'expliquer les formes de courbes représentées sur la figure 3 . Le troisième type d'interprétation consiste à faire intervenir une dispersion de la hauteur de barrière sur l'aire de la jonction $[18,19]$. Ceci est illustré par la figure 6 qui montre un réseau de courbes expérimentales obtenues sur Au-GaAs clivé sous ultra-vide à différentes températures [15]. On observe une apparition progressive de la forme « en $\mathrm{N}$ » quand la température diminue. Ceci est expliqué

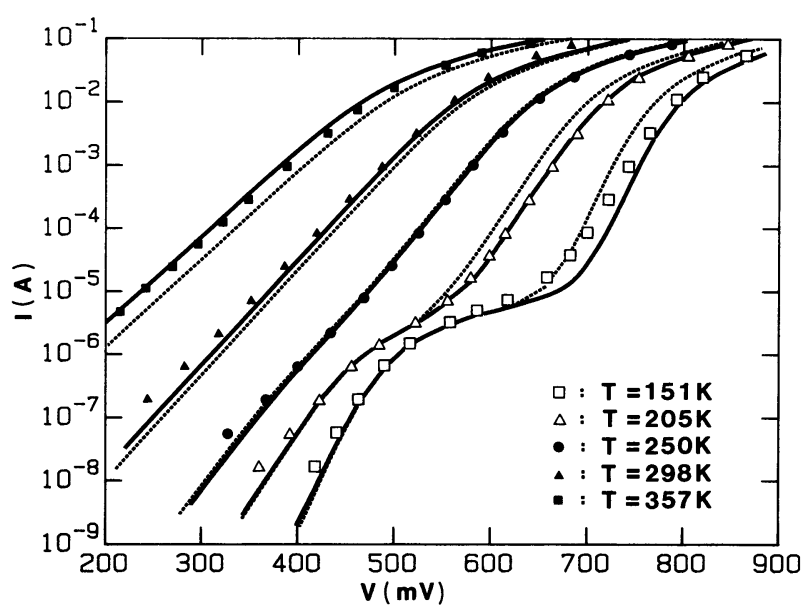

Fig. 6. - Caractéristiques $I-V$ à différentes températures pour $\mathrm{Au}-\mathrm{GaAs} \mathrm{N}$ clivé montrant une anomalie « en $\mathrm{N}$ ». Ce comportement est interprété ici par la présence de deux zones de conduction modélisables par deux diodes $D_{0}$ et $D_{1}$ en parallèle. Les courbes en pointillé sont calculées avec $\phi_{\mathrm{B}_{0}}=0,92 \mathrm{eV} ; \quad r_{0}=1,35 \Omega$; $\phi_{\mathrm{B}_{1}}=0,56 \mathrm{eV} ; r_{1}=2 \times 10^{4} \Omega$. Les courbes en trait plein tiennent en plus compte d'une dépendance à la température des hauteurs de barrière égale à celle du gap.

[ $I-V$ characteristics of an Au-GaAs N UHV cleaved contact showing an "N shape " anomaly. This behaviour is interpreted here by the existence of two conduction paths modelled by two diodes $D_{0}$ and $D_{1}$ in parallel. The dotted lines are calculated with $\phi_{\mathrm{B}_{0}}=0.92 \mathrm{eV} ; r_{0}=1.35 \Omega$; $\phi_{\mathrm{B}_{1}}=0.56 \mathrm{eV} ; r_{1}=2 \times 10^{4} \Omega$. The solid lines take into account a variation in barrier height equal to that of the band gap.] 
par la présence sur la diode de deux zones présentant chacune leur hauteur de barrière et leur résistance série particulières. Malgré la simplicité du modèle on constate un très bon accord avec les résultats expérimentaux.

Pour les courbes $C-V$ une étude de différentes perturbations envisageables a été effectuée par plusieurs auteurs [20,21]. Cependant, lorsqu'on analyse quantitativement le comportement en fonction de la tension, de la fréquence et de la température d'une courbe $C-V$ anormale il est très fréquent qu'aucune des solutions proposées ne soit applicable. C'est en particulier le cas pour les deux exemples de la figure 4. Une autre voie est de se livrer à une correction empirique. Deux solutions simples peuvent être envisagées : faire intervenir une capacité additionnelle soit en parallèle, soit en série. Le premier cas a été proposé par Vasudev [28] et une tentative d'interprétation basée sur la polarisabilité de GaAs a été fournie par Pellegrini [23]. Le second cas est évidemment typique d'une couche d'oxyde interfaciale. On devrait donc a priori attribuer la courbe $4 a$ à un mécanisme du premier type et la courbe $4 b$ à un effet de couche interfaciale. Mais là encore une analyse quantitative fait échouer les interprétations : si on corrige la courbe $4 a$ d'une capacité excédentaire on obtient un dopage beaucoup trop faible par rapport à la résistivité du matériau et pour expliquer la courbe $4 \mathrm{~b}$ à l'aide d'une capacité série il faudrait envisager une couche d'oxyde de plus de $100 \AA \AA$ d'épaisseur alors que des mesures par effet Auger montrent qu'elle est inférieure à trois couches atomiques. Nous montrerons au paragraphe 6 qu'une analyse fine de la forme de la barrière de potentiel peut fournir une nouvelle voie d'explication, valable dans les deux cas et quantitativement plausible.

\section{Etude des états d'interface.}

Nous rappelons succinctement le principe de la méthode de Spectroscopie de Capacité Schottky (SCS) qui est décrite en détail par ailleurs [24, 25]. Pour détecter un état d'interface il est nécessaire de modifier sa charge électrique. Cela est possible en perturbant une des probabilités d'échange de charges entre l'état et le semiconducteur ou le métal. Il est bien établi que dans une diode Schottky polarisée en direct, le quasi-niveau de Fermi des majoritaires se déplace dans le gap [16]. En d'autres termes la densité des porteurs majoritaires capables d'interagir avec les états d'interface dépend de la polarisation directe. En superposant un petit signal sinusoïdal à cette polarisation on provoque une modulation de la probabilité d'échange de charges entre les états et la bande de conduction (valence) du semiconducteur $\mathrm{s}$ 'il est de type $\mathrm{n}(\mathrm{p})$. Une capacité excédentaire $C_{\text {is }}$ va alors s'ajouter à la capacité de la zone de charge d'espace. $C_{\text {is }}$ dépend de la fréquence, de la température et de la polarisation par l'intermédiaire du temps de relaxation des états et de leur fonction d'occupation.

Plusieurs techniques expérimentales peuvent être utilisées [27, 28]. Nous présentons ici quelques résultats obtenus à l'aide de la méthode de balayage en température - ou SCS-T - récemment décrite [28]. Dans ce mode de fonctionnement, à un état d'énergie $E_{\mathrm{s}}$ correspond un pic de $C_{\text {is }}$ à une température $T_{\mathrm{p}}$ telle que :

$$
\frac{1}{K T_{\mathrm{p}}}=\frac{1}{E_{\mathrm{c}}-E_{\mathrm{s}}} \ln \frac{c_{\mathrm{n}} N_{\mathrm{c}}}{\left[4 \pi^{2} f^{2}+c_{\mathrm{n}}^{2} n_{\mathrm{S}}^{2}\right]^{1 / 2}}
$$

où $c_{\mathrm{n}}$ est le coefficient de capture des électrons, $n_{\mathrm{s}}$ leur densité à l'interface, $N_{\mathrm{c}}$ la concentration équivalente dans la bande de conduction et $f$ la fréquence du signal sinusoïdal. La figure 7 montre les résultats obtenus pour une interface Ag-GaAs n à différentes fréquences. A $100 \mathrm{kHz}$ la capacité mesurée est pratiquement confondue avec celle de la zone de charge d'espace. Mais quand la fréquence

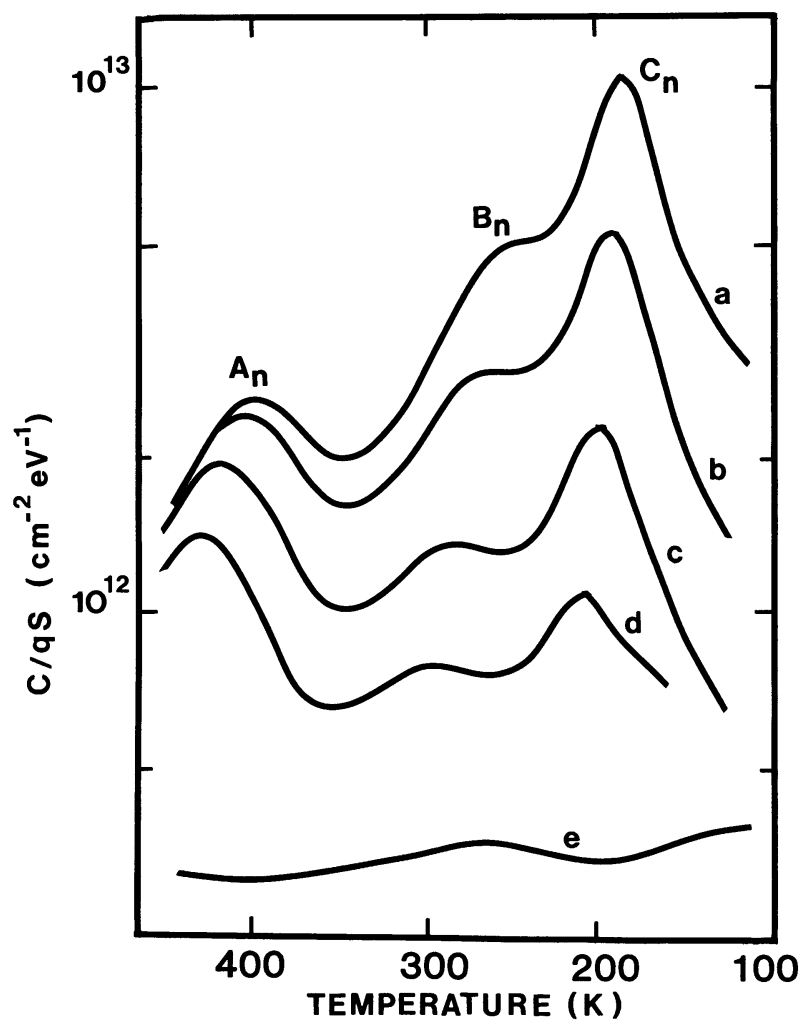

Fig. 7. - Variation de la capacité d'une diode AgGaAs $\mathrm{N}$ clivé sous vide en fonction de la température à différentes fréquences: a) $120 \mathrm{~Hz}$; b) $220 \mathrm{~Hz}$; c) $500 \mathrm{~Hz}$; d) $1 \mathrm{kHz}$; e) $100 \mathrm{kHz}$ [29].

[Variation in the capacitance of an Ag-GaAs N UHV cleaved diode as a function of temperature for various

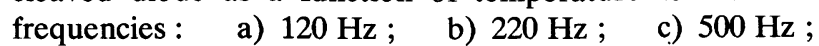
d) $1 \mathrm{kHz}$; e) $100 \mathrm{kHz}$ [29].] 
diminue on voit apparaître trois pics dus à trois états d'interface $A_{n}, B_{n}, C_{n}$ et dont l'intensité augmente progressivement. Lorsque l'amplitude d'un pic sature, la quantité $C_{\text {is }} / q S$ donne directement la densité des états. On constate aussi que ces pics glissent vers les basses températures lorsque $f$ diminue, conformément à l'équation (2). Tant que $f$ est supérieure à une fréquence critique $f_{\mathrm{c}}=c_{\mathrm{n}} n_{\mathrm{s}} / 2 \pi$ une courbe de $\left(k T_{\mathrm{p}}\right)^{-1}$ en fonction de $\ln \left(f \times\left(300 / T_{\mathrm{p}}\right)^{2}\right)$ est une droite dont la pente donne la position énergétique de l'état. De plus le coefficient de capture des électrons peut être déduit de l'intersection avec l'axe des fréquences. Il est essentiel de remarquer que cette droite n'est définie que par des paramètres caractéristiques de l'état d'interface. Elle en constitue une signature qui permet de détecter la présence d'un même état dans plusieurs interfaces différentes. Ceci est mis en évidence dans la figure 8 qui montre la signature du

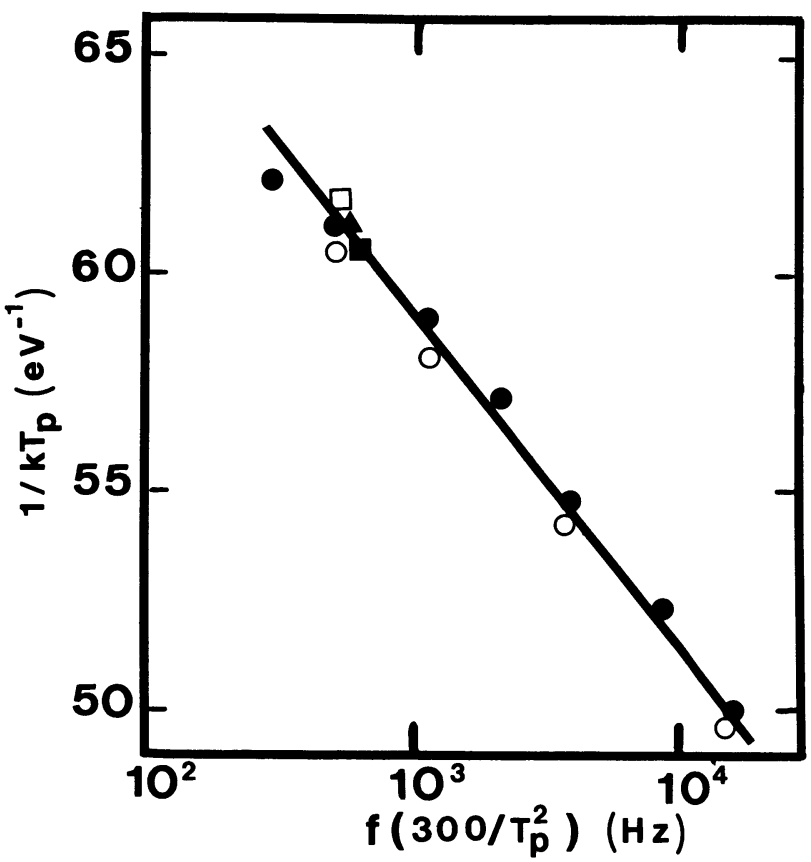

Fig. 8. - Signature de l'état $C_{\mathrm{n}}$ observé sur diverses interfaces $\mathrm{M}-\mathrm{GaAs}: \bullet \mathrm{Au}-\mathrm{GaAs}$ clivé sous vide ; $O$ idem après traitement thermique $250^{\circ} \mathrm{C}, 25 \mathrm{~min}$; Ag-GaAs clivé sous vide; $\square \mathrm{Ag}-\mathrm{GaAs}$ nettoyé chimiquement ; $\triangle \mathrm{Al}-\mathrm{GaAs}$ clivé sous vide. La pente de la droite donne l'énergie de l'état $\left(E_{\mathrm{c}}-0,30 \mathrm{eV}\right)$ et l'intersection avec l'axe des fréquences donne la section efficace de capture des électrons $\left(\sigma_{\mathrm{n}}=3 \times 10^{-14} \mathrm{~cm}^{2}\right)[29]$.

[Signature of the peak $C_{\mathrm{n}}$ observed on various GaAs diodes: - Au-GaAs UHV cleaved; $O$ idem after heat treatment $250^{\circ}, 25 \mathrm{~min} ; \mathrm{Ag}-\mathrm{GaAs} \mathrm{UHV}$ cleaved; $\square$ Ag-GaAs chemically etched; $\Delta$ Al-GaAs UHV cleaved. The slope of the straight line gives the energy level of the states $\left(E_{\mathrm{c}}-0.30 \mathrm{eV}\right)$ and the intersection with the frequency axis gives their electron capture crosssection $\left(\sigma_{\mathrm{n}}=3 \times 10^{-14} \mathrm{~cm}^{2}\right)[29]$.] pic $C_{\mathrm{n}}$ obtenue pour différents contacts $\mathrm{M}-\mathrm{GaAs}$. Plus généralement une comparaison qualitative directe peut être effectuée entre diverses interfaces en traçant une courbe $C_{\text {is }}(T)$ à la même fréquence pour chaque diode. Un état commun apparaîtra comme un pic à une même température, l'amplitude du pic étant proportionnelle à la densité de l'état à condition que les sections efficaces de capture des porteurs soient conservées. Sur la figure 9 sont ainsi comparés des spectres obtenus pour $\mathrm{Au}, \mathrm{Ag}$ et $\mathrm{Al}$ sur GaAs $n$ et $p$ [29]. Pour une meilleure compréhension l'échelle des températures est convertie en une échelle d'énergie permettant de situer approximativement les états dans le gap. On observe sur cette figure que les mêmes pics apparaissent quel que soit le métal avec diverses intensités. Ils correspondent donc à des états d'interface caractéristiques du semiconducteur mais dont la densité est modulée par la nature du métal. De plus on constate que la position des deux pics notés An et Ap est très proche

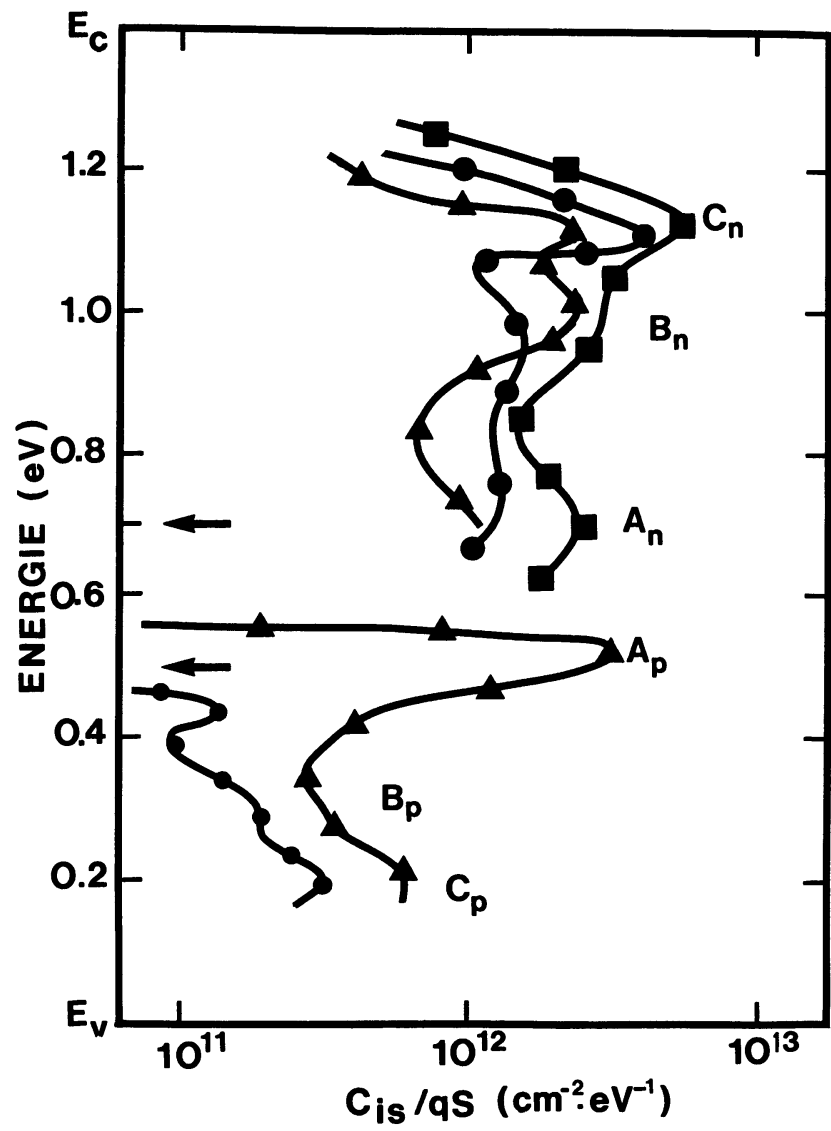

Fig. 9. - Capacité due aux états d'interface pour des contacts $\mathrm{Au}(\bullet), \mathrm{Ag}(\boldsymbol{\square}), \mathrm{Al}(\boldsymbol{\Delta})$ sur $\mathrm{GaAs}$ clivé sous vide [29]. Les flèches indiquent la position des niveaux de défauts de la théorie de Spicer [30].

[Interface state capacitance versus energy for $\mathrm{Au}(\bullet)$, $\mathrm{Ag}(\mathbf{\square}), \mathrm{Al}(\mathbf{\Delta})-\mathrm{GaAs}$ cleaved diodes [29]. Arrows indicate the location of the defect levels proposed by Spicer et al. [30].] 
des positions estimées par Spicer [30] pour les défauts responsables de l'ancrage du niveau de Fermi sur GaAs. Ces résultats confirment donc le « modèle unifié de défaut » mais indiquent que la densité de ces défauts est fonction de la nature du métal, peut être en liaison avec sa réactivité comme le suggère Brillson [31].

\section{Modélisation de la barrière de potentiel.}

Un autre point essentiel est apporté par les expériences de Spectroscopie de Capacité Schottky. L'étude détaillée du comportement de $C_{\text {is }}$ en fonction de la polarisation, la fréquence et la température montre que, dans la plupart des cas, les états observés sont peuplés préférentiellement par les porteurs majoritaires du semiconducteur. Ceci est vrai non seulement pour $\mathrm{GaAs}$ mais aussi pour $\mathrm{Si}[32,33]$ et InP [34]. Ceci implique que ces états s'étendent dans le semiconducteur suffisamment loin du métal pour que la probabilité de transfert d'électrons par effet tunnel soit négligeable. Compte tenu des estimations connues pour cette probabilité [35] on peut dire que cette extension doit être de quelques dizaines d'angströms au moins. Partant de ce fait on peut alors modéliser la barrière de potentiel au voisinage de l'interface en supposant la présence de quelques états, discrets en énergie comme le suggère la figure 9 et dont on considérera que la densité décroît exponentiellement à partir de l'interface suivant une loi du type :

$$
N_{\text {is }}(x)=K / L \exp (-x / L)
$$

où $K$ est la concentration totale de l'état et $L$ sa longueur caractéristique d'atténuation.

Quelques résultats caractéristiques sont indiqués sur la figure 10 pour différentes combinaisons d'états accepteurs et donneurs [36]. Ils étendent et précisent les estimations de hauteurs de barrière associées à une charge interfaciale légèrement enfouie sous la surface du semiconducteur récemment publiées [41,42] en montrant que la structure des bandes dans la zone de la barrière peut être extrêmement complexe et surtout que le sommet de la barrière peut être assez profondément "enterré " dans le semiconducteur. Ceci est aisé à comprendre en raisonnant sur le cas très simple d'un seul état accepteur dans un matériau de type $\mathrm{N}$. Le sommet de la barrière se produit à une distance $d$ de l'interface telle que la charge négative due à l'état et située au-delà de $d$ soit égale à la charge positive due aux dopants ionisés. La « couche-tampon " entre 0 et $d$ joue un rôle essentiel dans les propriétés de la jonction qui sera détaillée dans une autre publication [37]. Nous nous intéresserons seulement ici aux conséquences sur les caractéristiques électriques de base $C-V$ et $I-V$ [36].
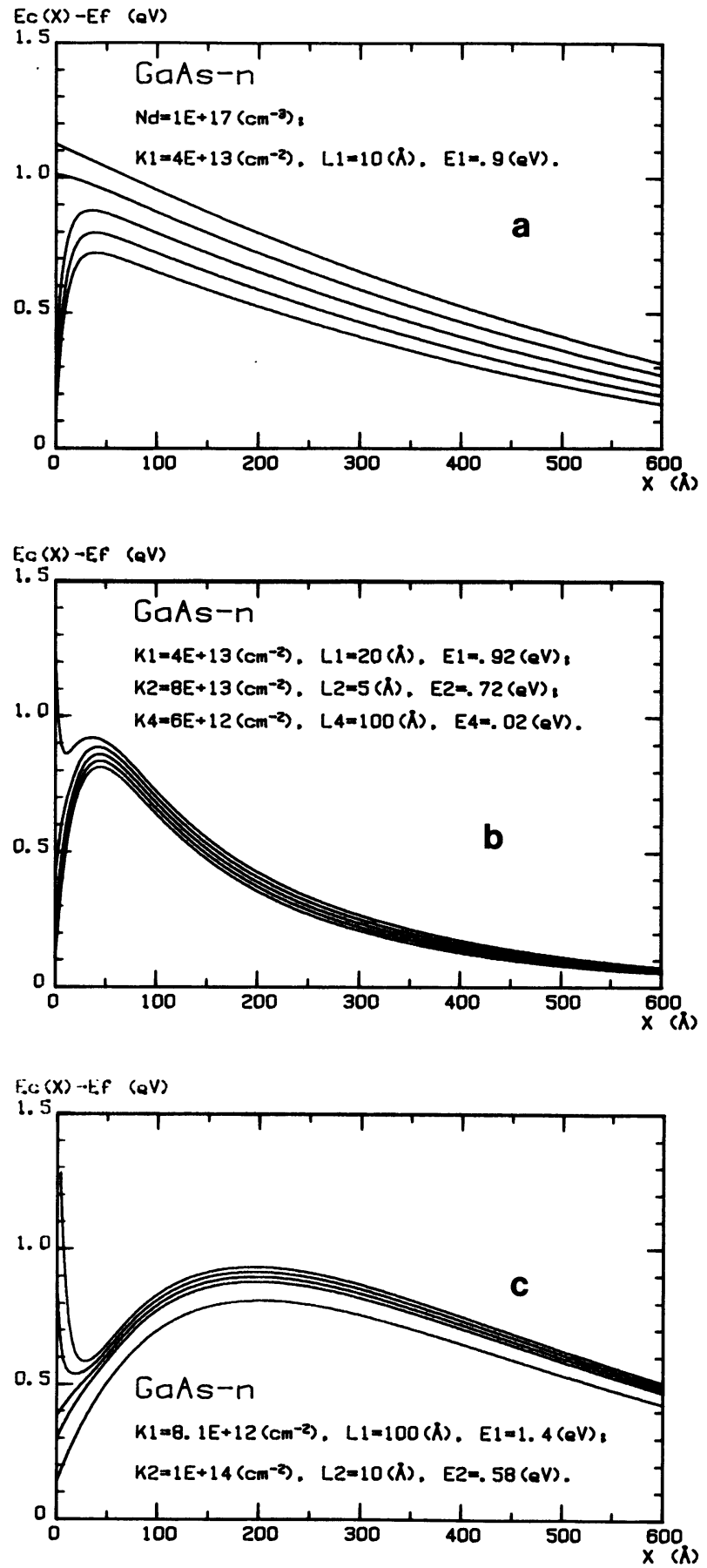

Fig. 10. - Profil de la bande de conduction dans le semiconducteur. Chaque réseau est obtenu pour une distribution d'états d'interface donnée en faisant varier le travail de sortie du métal : a) un état accepteur unique, b) les états 1 et 3 sont accepteurs, 2 est donneur, c) l'état 1 est accepteur, 2 est donneur. $E$ est la position énergétique de l'état par rapport à $E_{\mathrm{c}}, K$ est sa concentration totale et $L$ sa profondeur caractéristique de pénétration.

[Shape of the semiconductor conduction band. Each set of curves is obtained for a given distribution of interface states and various metal work function : a) an acceptor state, b) 1 and 3 are acceptor states ; 2 is a donor state, c) 1 is an acceptor state ; 2 is a donor state. $E$ is the energy level of the state (from $E_{\mathrm{c}}$ ), $K$ its density and $L$ its characteristic length of penetration in the semiconductor.] 
6. Conséquences sur les caractéristiques $C-V$ et $I-V$.

6.1 Caractéristiques $C-V$. - Il s'agit de considérer le rôle des états d'interface uniquement par leur influence électrostatique sur la forme de la barrière de potentiel et nullement par leur comportement dynamique comme au paragraphe 4. Nous nous plaçons donc dans des conditions de polarisation (inverse) et de fréquence (élevée) éliminant toute réponse directe des états. La figure 11 donne des résultats de simulation obtenus pour des interfaces M-GaAs en tenant compte d'un sommet de barrière enterré à la profondeur $d$. Comme on peut le voir, ceci provoque une caractéristique $C-V$ anormale présentant tous les aspects des résultats expérimentaux de la figure 4 : non-linéarité, barrière apparente trop élevée, dopage apparent trop faible. Le rôle de la couche-tampon peut se comprendre de la façon suivante : si la densité des états d'interface est suffisamment élevée, la variation de charge avec la polarisation dans la zone de déplétion est compensée par une modification très faible de $d$. En première approximation on peut considérer que $d$ est une constante. La couche-tampon se comporte alors comme une capacité $C_{d}$ constante en série avec la capacité de la zone de charge d'espace au-delà de

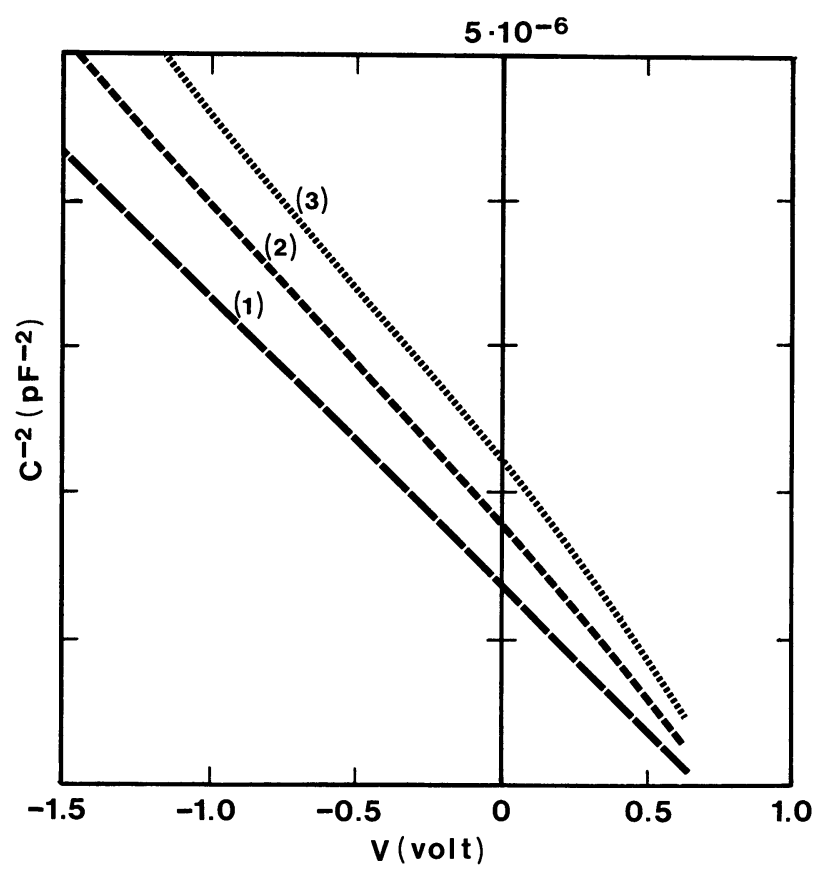

Fig. 11. - Courbes $C^{-2}-V$ obtenues en tenant compte d'un sommet de barrière situé à la profondeur $d$ dans le semiconducteur. Les courbes correspondent à GaAs type $\mathrm{N} ;\left(N_{\mathrm{D}}=1,6 \times 10^{17} \mathrm{~cm}^{-3} ; V_{\mathrm{D}}=0,7 \mathrm{~V}\right)$ et $d=0(1)$ $d=100 \AA$ (2) ; $d=200 \AA$ (3).

$\left[C^{-2}-V\right.$ curves corresponding to a barrier buried at a depth $d$ into the semiconductor. The curves are for $\mathrm{n}$ type $\mathrm{GaAs}$ $\left(N_{\mathrm{D}}=1.6 \times 10^{17} \mathrm{~cm}^{-3} ; V_{\mathrm{D}}=0.7 \mathrm{~V}\right)$ and $d=0$ (1), $d=100 \AA$ (2) ; $d=200 \AA$ (3).] $d$ qui, elle, suit la loi quadratique classique. Nous pensons que ce phénomène peut expliquer bon nombre d'anomalies observées sur des interfaces clivées sous ultra-vide ou présentant une couche d'oxyde très mince. Il est bien connu en effet que pour $\mathrm{Au}-\mathrm{GaAs}$ (Fig. 4a) et bien d'autres interfaces métal-semiconducteur III-V la réactivité des matériaux provoque la présence d'une couche interfaciale d'épaisseur non négligeable [38]. Cette couche peut être partiellement confondue avec la couche-tampon de notre modèle.

6.2 CARACTÉRISTIQUeS $I-V$. - Là encore nous ne nous intéressons qu'à l'influence purement électrostatique de la charge interfaciale en négligeant tout phénomène dynamique d'effet tunnel [39] ou recombinaison [40] via les états d'interface. La figure 12 donne des résultats de simulations effectuées sur une interface ayant les caractéristiques d'un contact $\mathrm{M}-\mathrm{GaAs}$ de type $\mathrm{N}$. Les trois courbes correspondent à trois positions d'un état d'interface situé en dessous, au voisinage ou au-dessus du niveau de Fermi du métal. Cet état est supposé en équilibre avec le semiconducteur, c'est-à-dire que sa population est régie par la position du quasi-niveau de Fermi des électrons. Cette position, donc la charge interfaciale, varie avec la tension de polarisation appliquée. On constate que les trois cas présentés correspondent aux trois anomalies typiques vues sur la figure 3. Le plus aisé à comprendre est le troisième en supposant que l'état est, par exemple, accepteur. La zone à forte pente correspond à la charge de l'état qui modifie l'épaisseur de la zone

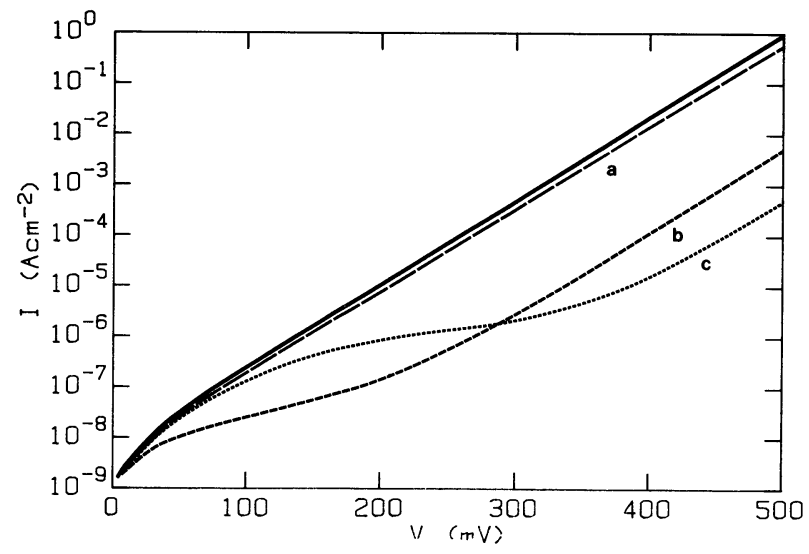

Fig. 12. - Simulation de courbes $I-V$ montrant les déformations introduites par l'effet électrostatique de la variation de charge d'un état d'interface accepteur. Les courbes correspondent à : a) état en dessous de $E_{\mathrm{FM}}$, b) état en $E_{\mathrm{FM}}$, c) état au-dessus de $E_{\mathrm{FM}}$.

[ $I-V$ curves showing the electrostatic effect of the interface charge on the thermoionic current. The curves are for an acceptor state located: a) below $E_{\mathrm{FM}}$; b) near $E_{\mathrm{FM}}$; c) above $E_{\mathrm{FM}}$.] 
tampon et la hauteur de barrière effective. Si l'état est au voisinage du niveau de Fermi il est déjà partiellement chargé et la zone à faible pente commence dès $V=0$. Enfin, même si l'état est en dessous du niveau de Fermi, il n'est pas totalement plein à température ambiante et c'est la complétion de sa charge qui donne une pente supérieure à $k T / q$ dans la courbe $12 \mathrm{a}$.

\section{Conclusion.}

L'étude des interfaces métal-semiconducteur par des méthodes de caractérisation électrique a pour but :

i) de déterminer la hauteur de barrière à partir de mesures de courant ou de capacité ;

ii) d'obtenir des informations sur les états d'interface par des méthodes de spectroscopie capacitive.

Nous avons montré que les problèmes posés par l'interprétation des courbes $I-V$ et $C-V$ étaient loin d'être résolus en raison des écarts de comportement de ces caractéristiques par rapport aux modèles de base. Nous avons brièvement rappelé les différents types d'explications qui ont été proposés à ces anomalies et souligné la nécessité d'une analyse quantitative précise. Si certains exemples expérimentaux s'accommodent d'une interprétation simple (courant excédentaire non thermoionique, fluctuation de hauteur de barrière, etc.) ce n'est pas le cas général.

Par ailleurs les études effectuées sur les états d'interface ont mis en évidence, particulièrement sur GaAs, l'existence d'états quasi discrets en énergie mais ayant une extension significative vers le volume du semiconducteur. Une modélisation développée sur ces bases montre que la forme des bandes d'énergie près de l'interface peut être très complexe. A l'aide de ce modèle et en se limitant aux cas les plus simples, il est possible de reproduire de façon satisfaisante les principaux comportements réels des caractéristiques électriques.

\section{Bibliographie}

[1] Cowley, M., Sze, S. M., J. Appl. Phys. 36 (1965) 3212.

[2] Mead, C. A., Solid-State Electron. 9 (1966) 1023.

[3] Kurtin, S., McGill, T. C., Mead, C. A., Phys. Rev. Lett. 22 (1970) 1433.

[4] Brillson, L. J., Phys. Rev. Lett. 40 (1978) 1978.

[5] Andrews, J. M., Phillips, J. C., Phys. Rev. Lett. 35 (1975) 56.

[6] Freeouf, J. L., Proc. 2nd Int. Conf. on Surf. and Interf. (Trieste) (1982) 233.

[7] Williams, R. H., Montgomery, V., Varma, R. R., J. Phys. C 11 (1978) 1989.

[8] Brillson, L. J., J. Vac. Sci. Technol. 15 (1978) 1378.

[9] Kendelewicz, T., Newman, N., List, R. S., LiNDAU, I., SPICER, W. E., J. Vac. Sci. Technol. B 3 (1985) 1206.

[10] Spicer, W. E., Lindau, I., Skeath, P., Su, C. Y., J. Vac. Sci. Technol. 17 (1980) 1019.

[11] Bolmont, D., Thèse, Université de Paris (1981).

[12] Newman, N., Spicer, W. E., Kendelewicz, T., LindaU, I., J. Vac. Sci. Technol. B 4 (1986) 931.

[13] Grant, R. W., Kraut, E. A., KowalczyK, S. P., WALDROP, J. R., J. Vac. Sci. Technol. B 1 (1983) 320.

[14] Tang, J. Y. F., Freeouf, J. L., J. Vac. Sci. Technol. B 2 (1984) 459.

[15] CheKir, F., Lu, G. N., Barret, C., Solid-State Electron. 29 (1986) 519.

[16] RHODERICK, E. H., IEEE Proc. 129 (1982) 1.

[17] Card, H. C., Rhoderick, E. H., J. Phys. D 4 (1971) 1589.

[18] Barret, C., Lebars, P., Vapaille, A., C.R. Hebd. Séan. Acad. Sci 280 (1975) 133.

[19] Freeouf, J. L., Woodall, M. J., Appl. Phys. Lett. 39 (1981) 727.

[20] Goodman, A. M., J. Appl. Phys. 34 (1963) 329.

[21] Fonash, S. J., J. Appl. Phys. 54 (1983) 1966.
[22] Vasudev, P. K., Mattes, B. L., Petras, E., Bube, R. H., Solid-State Electron. 19 (1976) 557.

[23] Pellegrini, B., Salardi, G., Solid-State Electron. 21 (1978) 465.

[24] Barret, C., Chekir, F., Vapaille, A., J. Phys. C 16 (1983) 2421.

[25] Muret, P., Deneuville, A., J. Appl. Phys. 53 (1982) 6289.

[26] Crowell, C. R., Beguwala, M., Solid-State Electron. 14 (1971) 1149.

[27] Chekir, F., Barret, C., Vapaille, A., J. Appl. Phys. 54 (1983) 6474.

[28] Chekir, F., Barret, C., Appl. Phys. Lett. 45 (1984) 1212.

[29] Chekir, F., Barret, C., Surf. Sci. 168 (1986) 838.

[30] SPicer, W. E., Eglash, S., Lindau, I., Su, C. Y., SKeATH, P., Thin Solid Films 89 (1982) 447.

[31] Brillson, L. J., Bruckner, C. F., Katnani, A. D., Stoffel, N. G., Margaritondo, G., Appl. Phys. Lett. 38 (1981) 784.

[32] Barret, C., Vapaille, A., J. Appl. Phys. 50 (1979) 4217.

[33] Muret, P., J. Appl. Phys. 53 (1982) 6300.

[34] Barret, C., Neffati, T., Chekir, F., à paraître.

[35] Muret, P., Thèse, Université de Grenoble (1984).

[36] Lu, G. N., Thèse, Université de Paris-Sud (1986).

[37] Lu, G. N., Barret, C., Neffati, T., à paraître.

[38] Brillson, L. J., Brucker, C. F., Margaritondo, G., Katnani, A. D., Stoffel, N. G., Phys. Rev. Lett. 46 (1981) 838.

[39] CARD, H. C., Solid-State Electron. 18 (1975) 881.

[40] Klimpke, C. M. H., LANDSberG, P. T., Solid-State Electron. 24 (1981) 401.

[41] Zur, A., McGill, T. C., Smith, D. L., Phys. Rev. B 28 (1983) 2060.

[42] Palau, J. M., Ismail, A., Lassabatère, L., SolidState Electron. 28 (1985) 499. 